\title{
Infant feeding practices of Caucasian women post partum living in Sheffield, UK
}

\author{
T. Mouratidou, F. A. Ford and R. B. Fraser \\ Reproductive and Developmental Medicine, Jessop Wing Hospital, University of Sheffield, Sheffield S10 2SF, UK
}

Evidence suggests that breast-fed babies are less likely to suffer from gastrointestinal infections and other long-term chronic conditions such as obesity and type 1 diabetes mellitus. Mothers who breast-feed also have a reduced risk in later life of some forms of cancer and osteoporosis. The WHO revised guidance on infant feeding suggests exclusive breast-feeding for the first 6 months of an infant's life and sets 6 months as the recommended age for the introduction of solid foods ${ }^{(1)}$.

The aim of the study was to establish how infants born to Caucasian mothers in Sheffield, UK are being fed and how maternal background characteristics might affect feeding practices as part of an on-going study evaluating the short-term effects of the introduction of 'Healthy Start' in pregnant women and women post partum and their newborns in Sheffield. Face-to-face (at 4 weeks post partum) and telephone (at 8,12,16, 20, 24, 36 and 46 weeks post partum) interviews were conducted with the new mothers using a subject information questionnaire and infant feeding questionnaires appropriately modified for each time point. Completed baseline data (at 4 weeks post partum) were provided by 155 Caucasian women with drop-out rates of $20-65 \%$ at each time point.

Mean maternal age was 26 years and mean BMI was $23 \mathrm{~kg} / \mathrm{m}^{2}$. Approximately $40 \%$ received food support, $69 \%$ were not in paid employment and $38 \%$ were self-reported smokers. Of the participants $67 \%$ intended to breast-feed based on individual influence. The Figure suggests that $60 \%$ of the infants were breast-fed initially (babies who were put to the breast, even if it was on one occasion only) and a decrease in the numbers of mothers breast-feeding with time post partum. The results of the regression analysis suggested that breast-feeding initiation was inversely affected by receipt of food benefits, maternal age, educational and smoking status. A comparison of the demographic and behavioural characteristics of mothers who were breast-feeding and formula-feeding also suggested education and income as strong predictors of breast-feeding initiation as well as past infant feeding practices and maternal meal patterns (Table).

\begin{tabular}{lll}
\hline & BF & FF \\
\hline Receipt of food support & 30 & $63^{*}$ \\
$\quad$ Yes & 70 & 37 \\
$\quad$ No & & \\
Previous infant feeding practices & 55 & $15^{*}$ \\
$\quad$ Breast-fed & 35 & 67 \\
Bottle-fed & 10 & 18 \\
$\quad$ Combination & & \\
Breakfast pattern & 64 & $42^{*}$ \\
Yes & 21 & 42 \\
No & 15 & 16 \\
$\quad$ Sometimes & & \\
\hline
\end{tabular}

$\mathrm{BF}$, breast-feeding; FF, formula feeding.

Mean values were significantly different from those for $\mathrm{BF}: * P=0.05$.

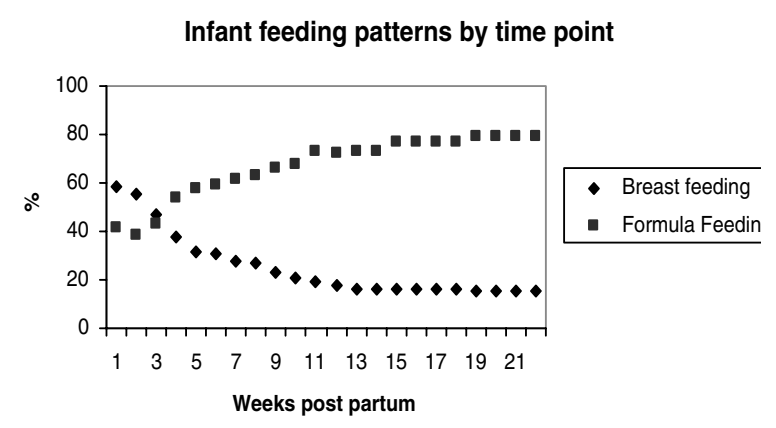

Similarly to other studies the results suggest a strong relationship between maternal socio-demographic and lifestyle characteristics and infant feeding practices ${ }^{(2,3)}$.

1. World Health Organization (2001) The Optimal Duration of Exclusive Breastfeeding: Report on an Expert Consultation. Geneva: WHO.

2. Kelly YJ \& Watt RG (2005) Public Health Nutr 8, 417-421.

3. Bolling K, Grant C, Hamlyn B \& Thornton A (2007) Infant Feeding Survey 2005. A Survey Conducted on Behalf of The Information Centre for Health and Social Care and the UK Health Departments by BMRB Social Research. London: The Information Centre. 